\title{
Correction to: In Vitro Seeding Activity of Glycoform-Deficient Prions from Variably Protease-Sensitive Prionopathy and Familial CJD Associated with PrP ${ }^{\mathrm{V} 180 \mathrm{l}}$ Mutation
}

\author{
Zerui Wang ${ }^{1,2}$. Jue Yuan ${ }^{2}$. Pingping Shen ${ }^{1} \cdot$ Romany Abskharon ${ }^{3} \cdot$ Yue Lang $^{1,2} \cdot$ Johnny Dang $^{2} \cdot$ Alise Adornato $^{2}$. \\ Ling $\mathrm{Xu}^{2}$. Jiafeng Chen ${ }^{1}$ • Jiachun Feng ${ }^{1}$ - Mohammed Moudjou ${ }^{4}$ - Tetsuyuki Kitamoto ${ }^{5}$. Hyoung-gon Lee ${ }^{6}$. \\ Yong-Sun Kim ${ }^{7}$. Jan Langeveld ${ }^{8}$ • Brian Appleby ${ }^{2,9,10}$ • Jiyan $\mathrm{Ma}^{3} \cdot$ Qingzhong Kong $^{2,9,10} \cdot$ Robert B. Petersen $^{2,11}$. \\ Wen-Quan Zou ${ }^{1,2,9,10,12}$ (D) Li Cui $^{1}$
}

Published online: 1 February 2019

(C) Springer Science+Business Media, LLC, part of Springer Nature 2019

\section{Correction to: Mol Neurobiol https://doi.org/10.1007/s12035-018-1459-0}

The original version of this article unfortunately contained a mistake.

The email address Dr. Wen-Quan Zou, one of the corresponding authors should be written as "wxz6@case.edu" instead of “wxz@case.edu”.

The authors need to add two more co-authors namely "Hyoung-gon Lee \& Yong-Sun Kim" as the 13th and 14th authors of this paper. This is because they had contributions to the generation of one of the transgenic mouse lines (TgV180I) used in this study. TgV180I mouse brain homogenates were used in the experiments along with other human and mouse brain homogenates.

The original article has been corrected.

Publisher's Note Springer Nature remains neutral with regard to jurisdictional claims in published maps and institutional affiliations.
The online version of the original article can be found at https://doi.org/ 10.1007/s12035-018-1459-0

\author{
Robert B. Petersen \\ robert.petersen@cmich.edu \\ $\triangle$ Wen-Quan Zou \\ wxz6@ case.edu \\ Li Cui \\ chuili1967@126.com
}

1 Department of Neurology, The First Hospital of Jilin University, Changchun, Jilin Province, People's Republic of China

2 Department of Pathology, Case Western Reserve University School of Medicine, Cleveland, OH, USA

3 Center for Neurodegenerative Science, Van Andel Research Institute, Grand Rapids, MI 49503, USA

4 Virologie Immunologie Moléculaires, INRA, Jouy-en-Josas, France

5 Center for Prion Diseases, Tohoku University Graduate School of Medicine, 2-1 Seiryo-machi, Aoba-ku, Sendai 980-8575, Japan
6 Department of Biology, College of Sciences, University of Texas at San Antonio, San Antonio, TX 78249, USA

7 Department of Microbiology, College of Medicine, Hallym University, Chuncheon, Gangwon-do 24252, Republic of Korea

8 Wageningen BioVeterinary Research, Houtribweg 39, Lelystad, the Netherlands

9 National Prion Disease Pathology Surveillance Center, Case Western Reserve University School of Medicine, Cleveland, OH, USA

10 Department of Neurology, Case Western Reserve University School of Medicine, Cleveland, OH, USA

11 Foundation Sciences, Central Michigan University College of Medicine, Mount Pleasant, MI, USA

12 State Key Laboratory for Infectious Disease Prevention and Control, National Institute for Viral Disease Control and Prevention, China Center for Disease Control and Prevention, Beijing, China 